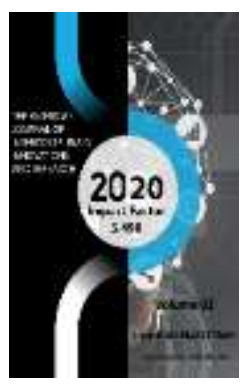

\title{
Connection Between A Right Triangle And An Equal Side Triangle
}

\author{
Ibragimov Husniddin Hikmatovich, \\ Lecturer Denau Institute Of Entrepreneurship And Pedagogy, Uzbekistan
}

Journal Website: http://usajournalshub.c om/index,php/tajiir

Copyright: Original content from this work may be used under the terms of the creative commons attributes 4.0 licence.

\section{ABSTRACT}

There is some evidence that a right triangle and an equilateral triangle are related. Information about Pythagorean numbers is given. The geometric meaning of the relationship between right triangles and equilateral triangles is shown. The geometric meaning of the relationship between an equilateral triangle and an equilateral triangle is shown.

\section{KEYWORDS}

Pythagorean theorem, Pythagorean numbers, right triangle, equilateral triangle.

\section{INTRODUCTION}

Theorem 1. If $\mathrm{a}, \mathrm{b}$, and $\mathrm{c}$ are Pythagorean numbers, then the following equation holds for any $\mathrm{n}$.

$$
\begin{aligned}
& \quad(n-2)(c-a)^{2}+(b-(n-2)(c-a))^{2}+\left(\frac{(n-1)(n-2)}{2}(c-a)+a-(n-2) b\right)^{2}= \\
& =\left(\frac{(n-1)(n-2)}{2}(c-a)+c-(n-2) b\right)^{2}
\end{aligned}
$$


Proof:

If $n=2$, then (1) equality $b^{2}+a^{2}=c^{2} \quad$ Takes the form of the Pythagorean theorem. Now let $n$ be an arbitrary number, then

$$
\begin{gathered}
(n-2)(c-a)^{2}+(b- \\
-(n-2)(c-a))^{2}+\left(\frac{(n-1)(n-2)}{2}(c-a)-(n-2) b+a\right)^{2}= \\
=\left(\frac{(n-1)(n-2)}{2}(c-a)-(n-2) b+c\right)^{2}
\end{gathered}
$$

We will open parentheses,

$$
\begin{gathered}
(n-2)(c-a)^{2}+b^{2}-2 b(n-2)(c-a)+((n-2)(c-a))^{2}+ \\
+\left(\frac{(n-1)(n-2)}{2}(c-a)-(n-2) b\right)^{2}+2 a\left(\frac{(n-1)(n-2)}{2}(c-a)-(n-2) b\right)+a^{2}= \\
=\left(\frac{(n-1)(n-2)}{2}(c-a)-(n-2) b\right)^{2}+2 c\left(\frac{(n-1)(n-2)}{2}(c-a)+(n-2) b\right)+c^{2}
\end{gathered}
$$

And we get the equation. The equation is the same on both sides $\left(\frac{(n-1)(n-2)}{2}(c-a)-(n-2) b\right)^{2}$ as a result we omit the expressions

$$
\begin{gathered}
(n-2)(c-a)^{2}+b^{2}-2 b(n-2)(c-a)+((n-2)(c-a))^{2}+ \\
+2 a\left(\frac{(n-1)(n-2)}{2}(c-a)-(n-2) b\right)+a^{2}= \\
=2 c\left(\frac{(n-1)(n-2)}{2}(c-a)-(n-2) b\right)+c^{2}
\end{gathered}
$$

There will be equality. $a, b$ and $c$ 's Pythagorean numbers are on the left side of the equation $a^{2}+b^{2}$ expression on the right $c^{2}$ from equality

$$
\begin{gathered}
(n-2)(c-a)^{2}-2 b(n-2)(c-a)+((n-2)(c-a))^{2}+2 a\left(\frac{(n-1)(n-2)}{2}(c-a)-(n-2) b\right) \\
= \\
=2 c\left(\frac{(n-1)(n-2)}{2}(c-a)-(n-2) b\right)
\end{gathered}
$$


Move the ending on the left side of the equation to the right side of the equation

$$
\begin{gathered}
(n-2)(c-a)^{2}-2 b(n-2)(c-a)+((n-2)(c-a))^{2}= \\
=2 c\left(\frac{(n-1)(n-2)}{2}(c-a)-(n-2) b\right)-2 a\left(\frac{(n-1)(n-2)}{2}(c-a)-(n-2) b\right)
\end{gathered}
$$

We will have. If on the right $2\left(\frac{(n-1)(n-2)}{2}(c-a)-(n-2) b\right)$ outside the parentheses of the total multiplier

$$
\begin{gathered}
(n-2)(c-a)^{2}-2 b(n-2)(c-a)+((n-2)(c-a))^{2}= \\
=2\left(\frac{(n-1)(n-2)}{2}(c-a)-(n-2) b\right)(c-a)
\end{gathered}
$$

Let's create an equation. Divide the equation by both sides (c-a)

$$
(n-2)(c-a)-2 b(n-2)+(n-2)^{2}(c-a)=2\left(\frac{(n-1)(n-2)}{2}(c-a)-(n-2) b\right)
$$

\section{Equation or}

$(n-2)(c-a)-2 b(n-2)+(n-2)^{2}(c-a)=2(n-2)\left(\frac{(n-1)}{2}(c-a)-b\right)$ We get

Now we divide both sides of the equation by non-zero $(n-2)$

$$
(c-a)-2 b+(n-2)(c-a)=2\left(\frac{(n-1)}{2}(c-a)-b\right)
$$

or

$$
(c-a)-2 b+(n-2)(c-a)=(n-1)(c-a)-2 b
$$

Hence $(n-1)(c-a)=(n-1)(c-a)$ which is the same. The theorem is proved.

Now from Theorem $1 n$ we get results for some values of

$n=2$ For, $\quad b^{2}+a^{2}=c^{2} \quad$ Pythagorean theorem.

$n=3$ For, $\quad(c-a)^{2}+(a+b-c)^{2}+(c-b)^{2}=(2 c-a-b)^{2}$

$n=4$ For, $\quad(c-a)^{2}+(c-a)^{2}+(b+2 a-2 c)^{2}+(3 c-2 a-2 b)^{2}=(4 c-3 a-2 b)^{2}$ 
$n=5$ For, $\quad(c-a)^{2}+(c-a)^{2}+(c-a)^{2}+(b+3 a-3 c)^{2}+(6 c-5 a-3 b)^{2}=$

$$
=(7 c-6 a-3 b)^{2}
$$

$n=k+2$ For, $\quad k(c-a)^{2}+(b-k(c-a))^{2}+\left(\frac{k(k+1)}{2}(c-a)+a-k b\right)^{2}$

$$
=\left(\frac{k(k+1)}{2}(c-a)+c-k b\right)^{2}
$$

We have an infinite number of formulas like

Now from the formula in Theorem $1 n \mathrm{~s}(n=2,3,4,5)$ shows the geometric meaning of the results obtained

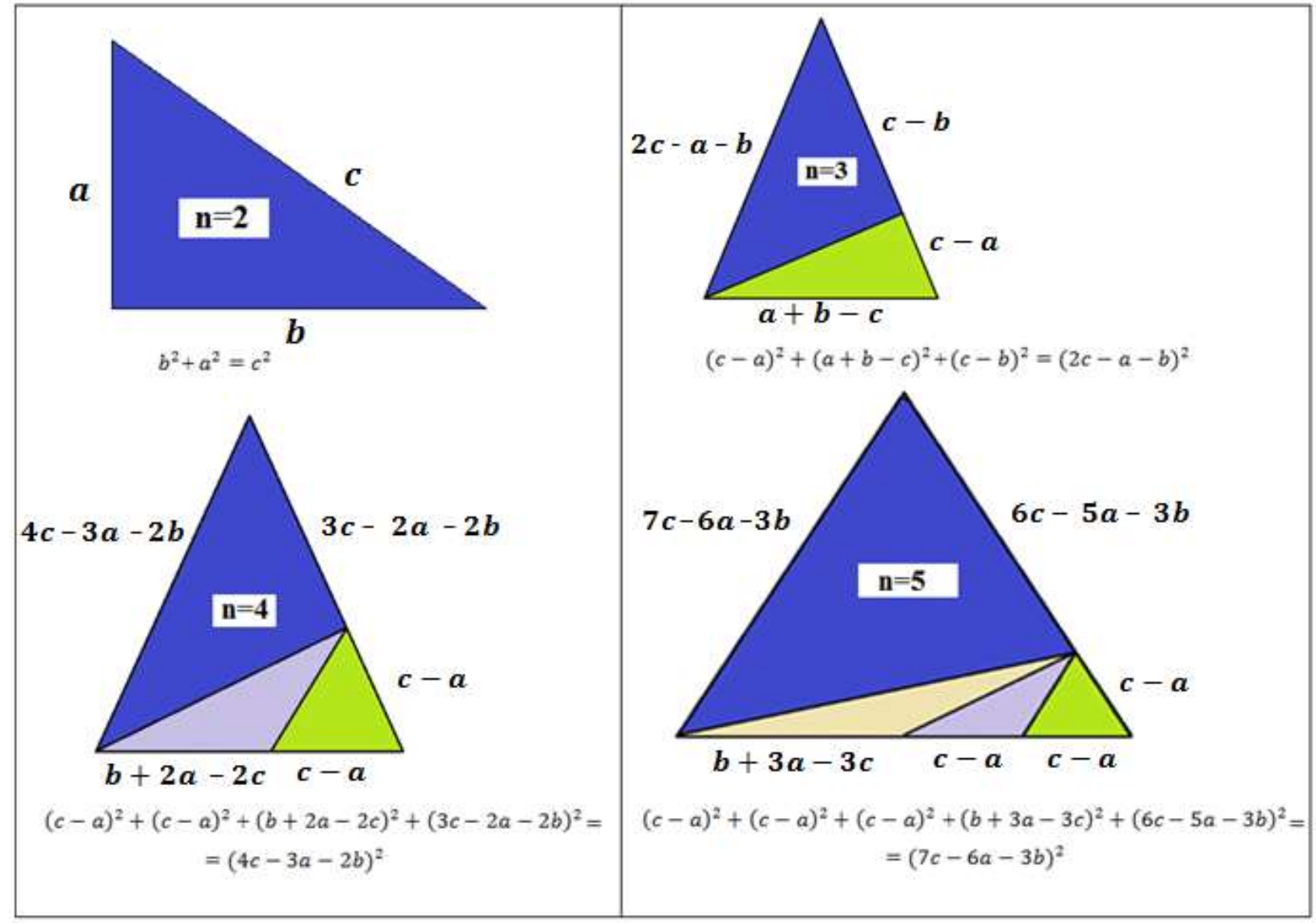

the values of Figure 1.

Figure 1 


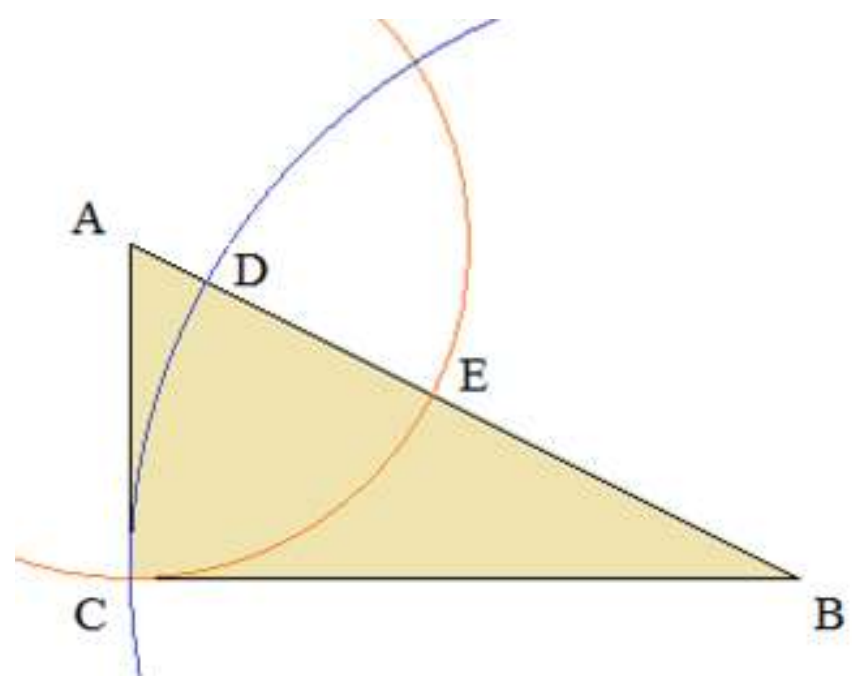

Figure 2

In Figure $1 n=2$ without $n=3$ what is the geometric method?

Let's say $A C$ the radius of the circle with the red line ( $A$ while the center of the circle) and $A C=b$, let it be, $B C$ and the radius of the circle with the green line ( $B$ while the center of the circle) $B C=a$ and $A B=C$ so to speak:

$A C=A E=b, \quad B C=B D=a, \quad A D=A B-D B=c-a$,

$B E=A B-A E=c-b, \quad D E=A B-A D-B E=c-(c-a)-(c-b)=a+b-c$,

$$
A D+B E=c-a+c-b=2 c-a-b
$$

We will have

Hence, we divide the hypotenuse of a right triangle into sections such as $-a, c-b$ and $a+b-c$ , as in Figure 2, and the sum of the two extreme sections $c-a$ and $c-b$ separated by the radii of the circles in the hypotenuse is for section $2 c-a-b:$

The result. $(c-a)^{2}+(a+b-c)^{2}+(c-b)^{2}=(2 c-a-b)^{2}$ equality is appropriate. This equation is in Theorem 1

$n=3$ that is to say.

Let us now consider the sequence of geometric transitions from $n=2$ to $n=3$ in Figure 1 . We only use a ruler and a compass. 


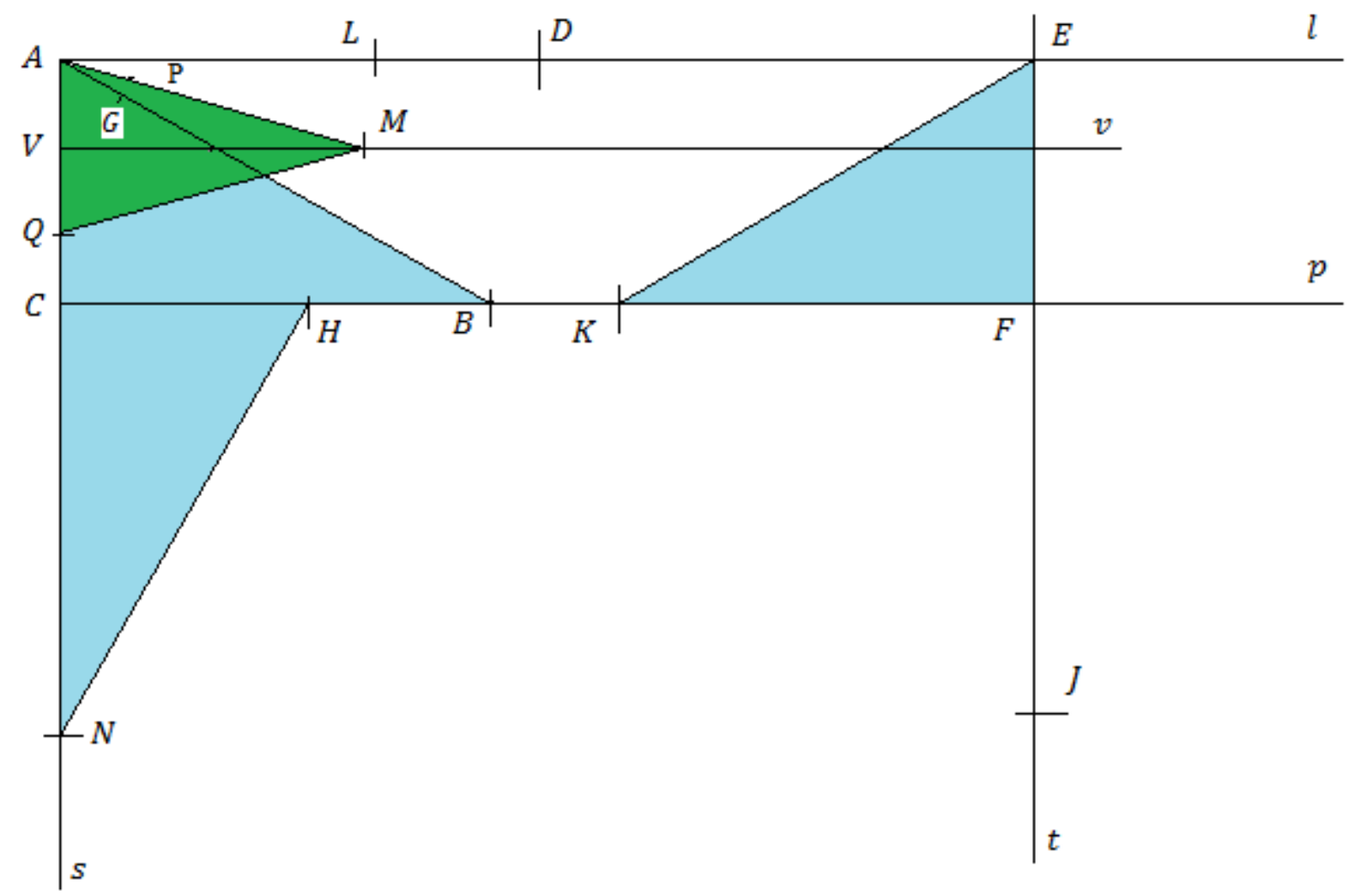

Figure 3

\section{THE MAIN FINDINGS AND RESULTS}

Let us have an arbitrary right triangle. We mark its ends with $A B C$ (see Figure 3). We agree that $A C=$ $b, B C=a$ and $A B=c$, respectively. We draw a straight line $p$ through the intersection $B C$. Draw a straight line $l$ parallel to the straight line $p$ from point $A$ perpendicular to the $A C$ section. It is known that $A B=c$. Using a compass, we denote $A B=A D=c$ by a straight line $l$ centered at the end $A$. Let $A D=D E=c$ be the center of the point $l$ on the straight line $l$, and we find $A E=2 c$. Now $l$ perpendicular to the straight line $E$ passing point $t$ t we cross the thief line. This straight line $p$ intersects the straight line and we denote this point by $F$. By construction, we can say $A C=E F=b$ . Using a compass, we define a point $K$ on a straight line $\mathrm{p}$ with the center of the end $D E$ and find $E K=$ $c$, then $K F=a . K F$ using a compass $F$ centering the tip $t$ in a straight line $J$ nand $F J=a$ we find in that case $E J=a+b$. Using a compass $E J$, we define a point $l$ on a straight line $l$ with the center of the end $E$, and $E L=a+b$. To us $A E=2 c$ was from it $E L=a+b$ if we separate it $A L=2 c-$ $a-b$ we find.

On the other hand $A C$ crossing $s$ Let's draw a straight line. $s$ in a straight line $B C$ using a compass. $C$ centering the tip $N$ define the point and $C N=a$. $A C$ using a compass $C$ centering the tip $p$ in a straight line $H$ define the point and $C H=b$ we find in that case $N H=c, A C=b$ va $C N=a$ 
because $N A=a+b$ arises. $N H$ using a compass $N$ centering the tip $s$ in a straight line $Q$ define the point and $N Q=c$ we find in that case $N A=a+b$ from $N Q=c$ lost $Q A=a+b-c$ found. Using a compass and a ruler, find the middle of the $Q A$ section and mark it with a $V$. Draw a straight line $v$ perpendicular to the straight line s from point $V$. Using a compass $A L$, center the end $A$ and mark the point $M$ on the straight line $v$ and find $A M=A L=2 c-a-b$.

In this case, $A M=M Q=2 c-a-b$. Using a compass, we mark the point $\mathrm{G}$ from the intersection $A B$ with the center $B$ as the center. Then $B C=B G=a \quad$ and $A B=c$ and $B G=a$, then $A G=c-a$. Using a compass, we define the point $\mathrm{P}$ from the intersection $A M$ with the center $A$ at the end $A$. Then $A P=A G=c-a \quad$ If $A M=2 c-a-$ and $A P=A G=c-a \quad$, then $P M=c-b$. U. And we have shown the geometric method of transition from the generalized formula of the Pythagorean theorem to the result of $n$ for $n=2$ for $n=3$ for a case where one end is common. (We have shown the geometric method for a right-angled triangle $A B C$ in the case $n=2$ from the equilateral triangle $A Q M$ in the case $n=3$ for the case where the end $A$ is common).

Let us now consider the sequence of geometric transitions from $n=3$ to $n=2$ in Figure 1. Of course, we use a ruler and a compass.

Let us be given an arbitrary equilateral triangle. We mark its ends with $A B C$ (see Figure 4). Suppose $A B=2 c-a-b, \quad A C=a+b-c, C T=c-b$ and $T B=c-a$ respectively.

Using a compass and a ruler, draw a straight line $l$ perpendicular to the end $A$ of the $A C$ section. It is known that $A B=2 c-a-b$. Using a compass, we denote $A B=A P=2 c-a-b$ by a straight line $l$ with the center $A$ at the end. Using a compass, we denote $A C=A E=a+b-c$ by a straight line $l$ centered on tip $A$. Then $A E+A P=E P=c \quad$ Let's draw a straight line $f$ intersecting $A C . \quad C T$ Using a compass $C$ centering the tip $f$ in a straight line $C T=C D=c-b$ we define. In this case $+C D=$ $A D=a$. Draw a straight line $f$ through the point $D$ and a straight line $p$ perpendicular to it. In this case, the straight lines $l$ and $p$ are perpendicular to each other. If we draw a line perpendicular to the point $E$ of a straight line $l$, this line intersects the straight line $p$ at right angles, we denote this point by $M$. Then $A D=E M=a$ Using a compass, we define $E P=E K=c$ on a straight line $\mathrm{p}$ with the center $\mathrm{E}$ as the center. Hence, $M K=b$. From point A we draw a straight line $v$ perpendicular to the section $E K$ and denote the point where this line intersects by $F$. If we draw a straight line $t$ from the point $A$ of the section $A F$ F perpendicular to the section itself, and denote by $\mathrm{N}$ the point where the straight line $t$ intersects the straight line $p$. In that case

Given that $E K=A P$ va $E M=A D$, we can say that $M K=D N$.

And we have shown the geometric method of transition from the generalized formula of the Pythagorean theorem to the result of $n$ for the value $n=3$ to the result obtained for the value of $n=$ 2 , where one end is common. (We have shown the geometric method for the case where the end $A$ is common to the right-angled triangle $\mathrm{ABC}$ in the case $n=3$ from $A B C$ to the right triangle $A B C$ in the case $n=2$ 


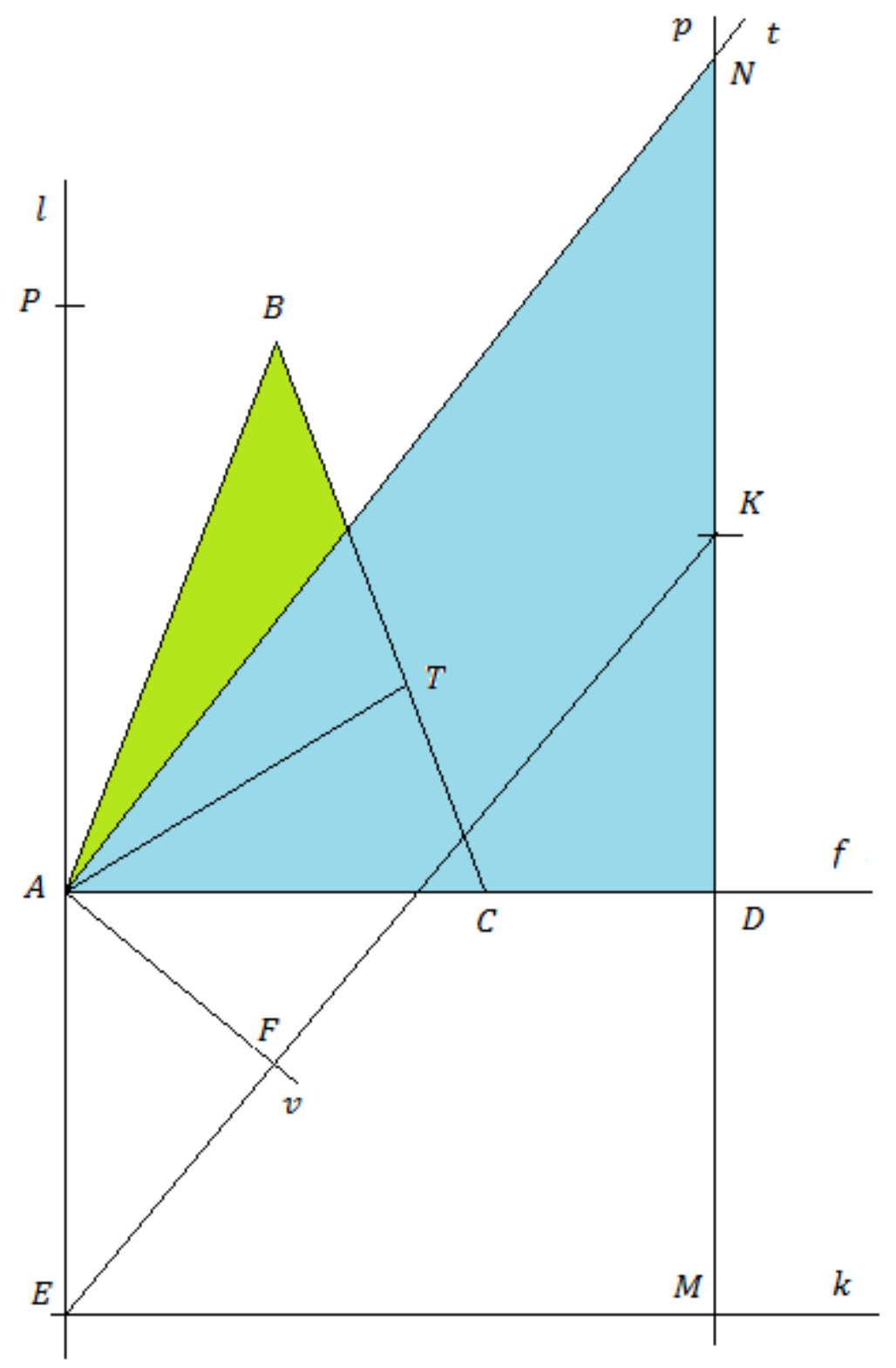

Figure 4

Above we

$$
\begin{aligned}
(n-2)(c-a)^{2}+(b- & (n-2)(c-a))^{2}+\left(\frac{(n-1)(n-2)}{2}(c-a)+a-(n-2) b\right)^{2}= \\
& =\left(\frac{(n-1)(n-2)}{2}(c-a)+c-(n-2) b\right)^{2}
\end{aligned}
$$


There are the following relationships between the results obtained for different values of $n$ in the formula

Conditionally in the formula $b-(n-2)(c-a)$ we can call the expression "compiler". In the formula $\frac{(n-1)(n-2)}{2}(c-a)+c-(n-2) b$ The expression is equal to the length of the side of the triangle and $\frac{(n-1)(n-2)}{2}(c-a)+a-(n-2) b$ Given that the expression is always less than $c-a$ from the side in an equilateral triangle, let us conditionally call it a "short section". Given that c-a is formed by adding $c-a$ to the short part of the formula, we can conditionally call $c-a$ a "filler".

In this case, the formula finds the parts of an equilateral triangle with number $n>3$ and the sides of an equilateral triangle with number $n-1$ with the following regularity. In the case of $n=3$, it is shot from an equilateral triangle into a right triangle.

The above-mentioned law is as follows.

a) If we add a filler to the compiler for $n$, we find the compiler of the case $n-1$, and the parts equal to the remaining $n-3$ fillers become $n-1$

b) $n$ We add a short fraction to the compiler in case $n$ and find the short fraction in case $n-1$.

c) Add a side to the compiler in case $n$ and find the side in case $n-1$.

\section{CONCLUSION}

We show in the diagram the transition from the first $n=3$ to the state $n=2 \quad$ using the abovementioned law (see Figure 5). Given the apparent nature of the diagram, we will not dwell on the sequence of application of the law.

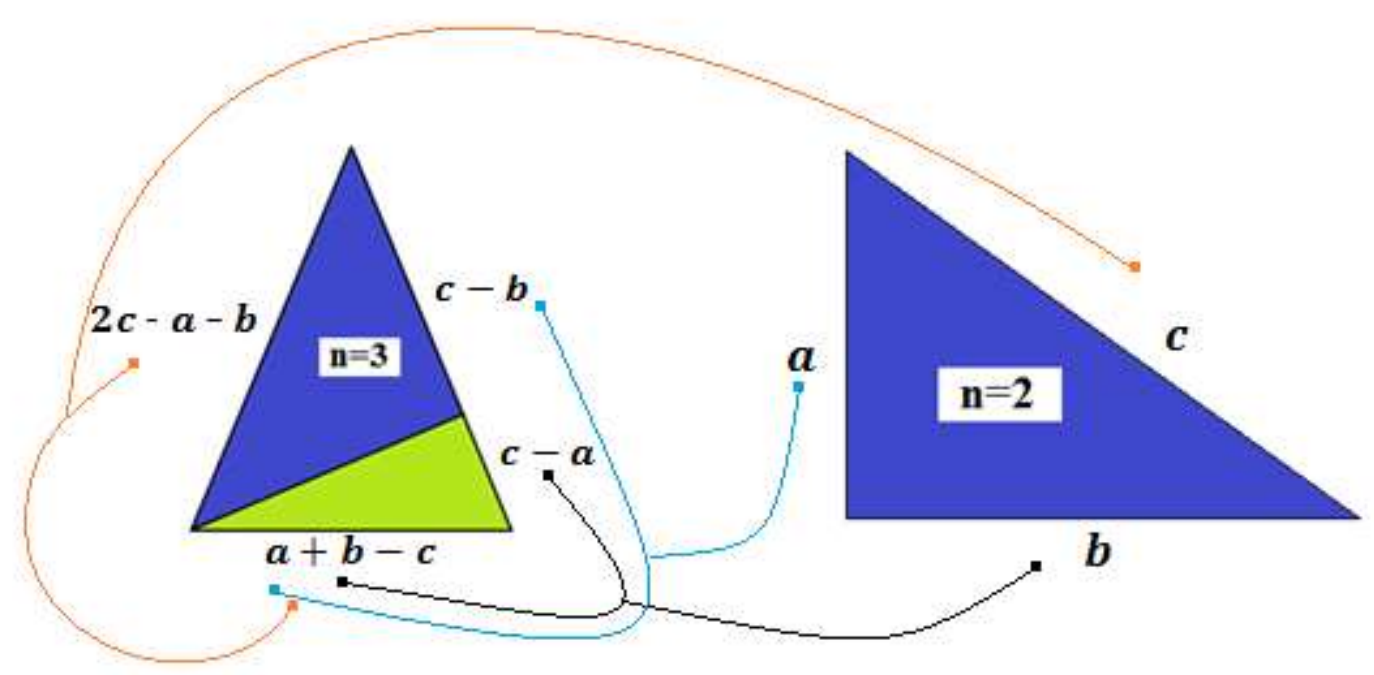

Figure 5 
As a second example $n>3$ using the above-mentioned law $=5, n=4$ We show the transition from state to state in the diagram. (Consider Figure 5). Given the apparent nature of the diagram, we will not dwell on the sequence of application of the law.

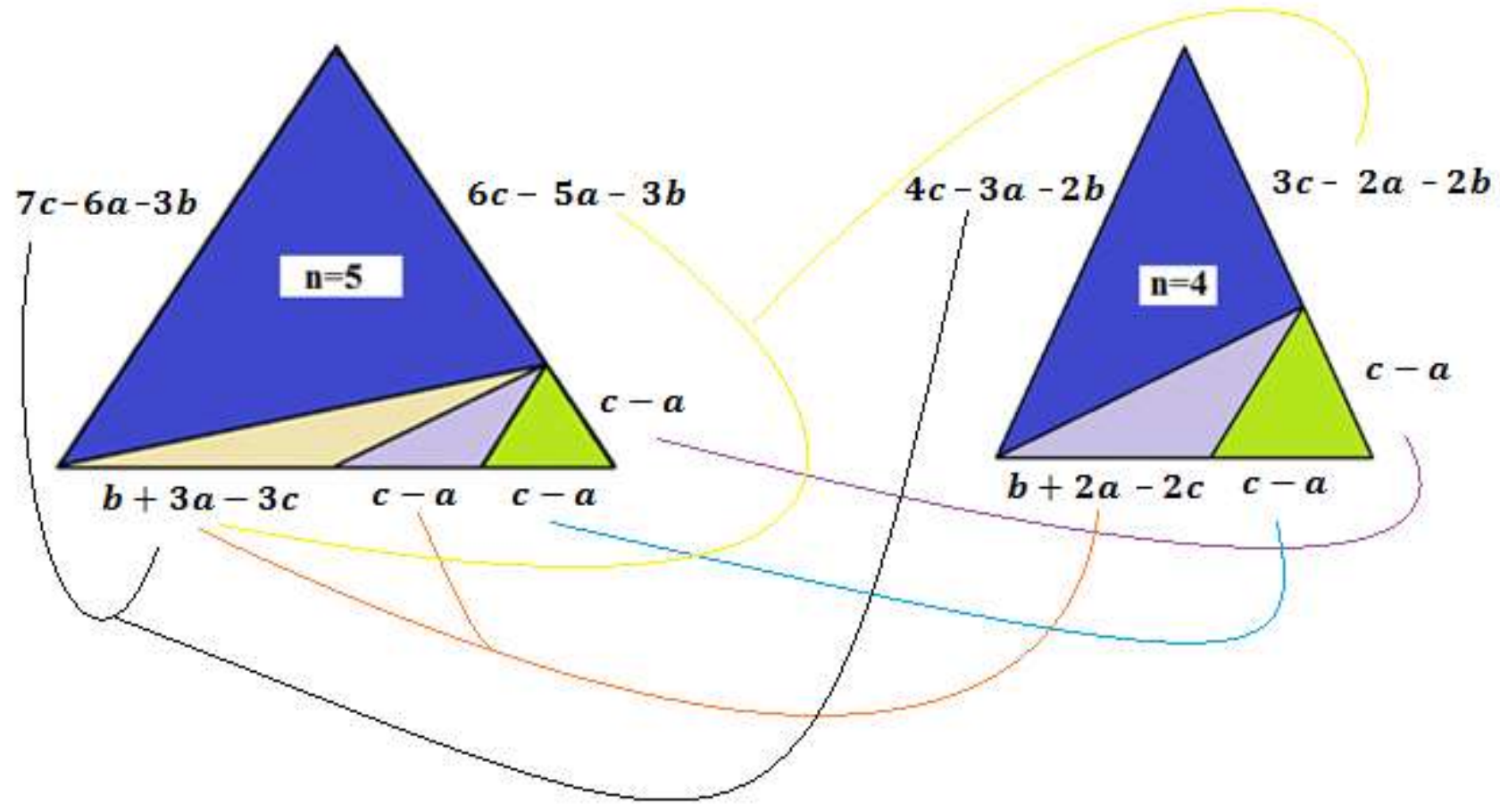

Figure 6

\section{REFERENCES}

1. A. Azamov. Euler bricks. Physics, mathematics and computer science. 2012.№1

2. R. Spira. The diophantine equation. Amer. Math. Monthly.1962. T. 69

3. Ian Stewart. Greatest math problems. M: Alpina non-fiction, 2016.

4. B.D. Sokolowsky, A. G. Van Hooft, R. M. Volkert, C. A. Reiter. Math. Comp. 83(2014), №. 289

5. A.V. Voron. A method for obtaining Euler parallelepipeds based on the cotangent values of Pythagorean triplets "Academy of Trinitarianism", Moscow: publ. 25656, 17.08.2019. 